\title{
Arginine-259 of UGT2B7 Confers UDP-Sugar Selectivity ${ }^{\llbracket}$
}

\author{
(1) Pramod C. Nair, ${ }^{1}$ Nuy Chau, ${ }^{1}$ Ross A. McKinnon, and John O. Miners
}

\begin{abstract}
Department of Clinical Pharmacology (P.C.N., N.C., J.O.M.) and Flinders Health and Medical Research Institute (FHMRI) Cancer Program (P.C.N., R.A.M., J.O.M.), Flinders Health and Medical Research Institute, Flinders University College of Medicine and Public Health, Flinders Medical Centre, South Australia, Australia
\end{abstract}

Received June 29, 2020; accepted September 17, 2020

\begin{abstract}
Enzymes of the human UDP-glycosyltransferase (UGT) superfamily typically catalyze the covalent addition of the sugar moiety from a UDP-sugar cofactor to relatively low-molecular weight lipophilic compounds. Although UDP-glucuronic acid (UDPGlcUA) is most commonly employed as the cofactor by UGT1 and UGT2 family enzymes, UGT2B7 and several other enzymes can use both UDP-GIcUA and UDP-glucose (UDP-Glc), leading to the formation of glucuronide and glucoside conjugates. An investigation of UGT2B7-catalyzed morphine glycosidation indicated that glucuronidation is the principal route of metabolism because the binding affinity of UDP-GIcUA is higher than that of UDP-Glc. Currently, it is unclear which residues in the UGT2B7 cofactor binding domain are responsible for the preferential binding of UDP-GIcUA. Here, molecular dynamics (MD) simulations were performed together with site-directed mutagenesis and enzyme kinetic studies to identify residues within the UGT2B7 binding site responsible for the selective cofactor binding. MD simulations demonstrated that Arg259, which is located within the
\end{abstract}

$\mathrm{N}$-terminal domain, specifically interacts with UDP-GlcUA, whereby the side chain of Arg259 $\mathrm{H}$-bonds and forms a salt bridge with the carboxylate group of glucuronic acid. Consistent with the MD simulations, substitution of Arg259 with Leu resulted in the loss of morphine, 4-methylumbelliferone, and zidovudine glucuronidation activity, but morphine glucosidation was preserved.

\section{SIGNIFICANCE STATEMENT}

Despite the importance of uridine diphosphate glycosyltransferase (UGT) enzymes in drug and chemical metabolism, cofactor binding interactions are incompletely understood, as is the molecular basis for preferential glucuronidation by UGT1 and UGT2 family enzymes. The study demonstrated that long timescale molecular dynamics (MD) simulations with a UGT2B7 homology model can be used to identify critical binding interactions of a UGT protein with UDP-sugar cofactors. Further, the data provide a basis for the application of MD simulations to the elucidation of UGT-aglycone interactions.

\section{Introduction}

Enzymes of the human UDP-glycosyltransferase (UGT) superfamily catalyze the transfer of a hexose moiety from a UDP-sugar to a nucleophilic acceptor functional group present in structurally diverse compounds that include drugs, nondrug xenobiotics, and endobiotics (Miners and Mackenzie, 1991; Radominska-Pandya et al., 1999; Miners et al., 2004; Mackenzie et al., 2005; Meech et al., 2019). This versatility of UGT arises from the existence of multiple forms of the enzyme that exhibit distinct but frequently overlapping substrate selectivities (Miners et al., 2010). The 22 functional human UGT genes identified to date are classified into four families: UGT1, UGT2, UGT3, and UGT8. Utilization

This study was supported by the National Health and Medical Research Council of Australia [Grant 1120137]. This research was undertaken with the assistance of resources from the National Computational Infrastructure (NCI), which is supported by the Australian Government. R.A.M. is a Beat Cancer Professorial Fellow supported by Cancer Council South Australia (SA) and SA Health.

${ }^{1}$ P.C.N. and N.C. are joint first authors.

https://doi.org/10.1124/molpharm.120.000104.

S This article has supplemental material available at molpharm. aspetjournals.org. of UDP-sugars by human UGTs varies between families and enzymes. For example, UGT3A1 and UGT3A2, the only members of the UGT3 family, use N-acetylglucosamine and UDP-glucose (UDP-Glc) as the respective cofactors, whereas UGT8A1 utilizes only UDP-galactose (Mackenzie et al., 2008; Meech et al., 2012, 2015). By contrast, enzymes of the UGT1 and UGT2 families primarily use UDP-glucuronic acid (UDP-GlcUA) as the cofactor, although some enzymes in these families are also known to catalyze glycosidation reactions other than glucuronidation (Meech et al., 2012). Nevertheless, glucuronidation is the major metabolic pathway since, at least with UGT2B7, the affinity of UDP-GlcUA is higher than that of the alternate UDP-sugar (Chau et al., 2014 and references therein). These observations suggest that differences in cofactor binding are responsible for the differing UDP-sugar selectivities of UGT enzymes.

UGT2B7 is considered to be one of the most important enzymes involved in drug glucuronidation. This enzyme contributes to the glucuronidation of drugs from numerous therapeutic classes that include opioids (e.g., codeine, morphine, naloxone), anticancer drugs (e.g., epirubicin), nonsteroidal anti-inflammatory agents (e.g., diclofenac, naproxen),

ABBREVIATIONS: 4MU, 4-methylumbelliferone; Aro, aromatic; AZT, zidovudine; CT, C-terminal; HEK, human embryonic kidney; MD, molecular dynamics; MDS, MD simulation; MEM, minimum essential media; NT, N-terminal; PCR, polymerase chain reaction; SDM, site-directed mutagenesis; UDP-Glc, UDP-glucose; UDP-GlcUA, UDP-glucuronic acid; UGT, UDP-glycosyltransferase. 
anticonvulsants (e.g., lamotrigine, valproic acid), antibiotics (e.g., chloramphenicol), and antiretroviral agents [e.g., zidovudine (AZT)] (Radominska-Pandya et al., 2001; Miners et al., 2010; Stingl et al., 2014). Morphine (3- and 6- glucuronidation) and AZT are the prototypic selective substrates of UGT2B7 (Court et al., 2003; Stone et al., 2003; Miners et al., 2010).

Notably, however, UGT2B7 also catalyzes the glucosidation of several drugs, including the 3-glucosidation of morphine (Chau et al., 2014). The respective cofactors for glucosidation and glucuronidation reactions are UDP-Glc and UDP-GlcUA. UDP-glucose dehydrogenase catalyzes the synthesis of UDPGlcUA from UDP-Glc by a two-stage $\mathrm{NAD}^{+}$-dependent reaction (Feingold and Franzen 1981), and hence both cofactors are available in hepatocytes for drug and chemical conjugation. However, it remains unclear which residues within the UGT2B 7 binding site contribute toward the higher binding affinity of UDP-GlcUA that favors glucuronidation over glucosidation.

The C-terminal (CT) domain is highly conserved across the human UGT family (Radominska-Pandya et al., 1999; Mackenzie et al., 2005). In particular, $\sim 45$ conserved residues, which are referred to the UGT signature sequence, located in the CT domain have been identified as a key region for UDPsugar binding from sequence alignment, chimeragenesis, and site-directed mutagenesis studies and from the X-ray crystal structures of plant UGTs (Mackenzie et al., 2005; Offen et al., 2006; Meech et al., 2012, 2015). Although an X-ray crystal structure of a full-length mammalian UGT protein is unavailable, partial structures of the CT domain of human UGT2B7 and UGT2B15 without bound UDP-sugar have been published (Miley et al., 2007; Zhang et al., 2020). Taken together, the data demonstrate that the UDP-sugar and substrate (aglycone) binding domains occur predominantly in the CT and N-terminal (NT) domains, respectively (Miley et al., 2007; Nair et al., 2015). However, the partial X-ray crystal structures and structure-function studies have only provided limited understanding into the UDP-sugar selectivity of human UGT enzymes. For instance, molecular modeling studies of the UGT2B7 protein identified CT domain residues (e.g., Asn378, Asn402) associated with preferential binding of UDP-GlcUA over UDP-Glc (Miley et al., 2007; Chau et al., 2014). However, mutagenesis of these amino acids led to loss of all glycosidation activity, which is inconsistent with a selective role in cofactor binding (Chau et al., 2014).

Homology models of several UGT enzymes have been developed in this laboratory to investigate the UDP-sugar selectivites of UGT2 (including UGT2B7), UGT3, and UGT8 family proteins (Nair et al., 2015). Homology models of the CT domain showed a highly conserved structural fold with respect to the UGT2B7-CT X-ray crystal structure [root-mean-square deviations (rmsds) between 0.066 and $0.079 \AA$ ] and predicted interactions consistent with those anticipated from the X-ray crystal structure (Miley et al., 2007). Based on a full-length homology model of UGT2B7, we postulated that residue(s) from the NT domain are important for UDP-sugar recognition and may play a significant role in the cofactor selectivities of UGT enzymes. However, the static models provided limited insight into how individual residues and their flexibility contributed toward UDP-sugar binding and selectivity. In this study, we used long molecular dynamics (MD) simulation (MDS) times together with site-directed mutagenesis and enzyme activity assays to demonstrate that Arg-259 of the
NT domain plays a pivotal role in the binding selectivity of UDP-GlcUA.

\section{Materials and Methods}

Chemicals and Other Reagents. 4-Methylumbelliferone (4MU), 4-methylumbelliferone glucuronide, morphine $3-\beta$-D-glucuronide, morphine 6 - $\beta$-D-glucuronide, 1-octanesulfonic acid sodium salt, UDP-Glc (disodium salt) from Saccharomyces cerevisiae, UDPGlcUA (trisodium salt), puromycin, ammonium persulfate, AZT, and zidovudine glucuronide were purchased from Sigma-Aldrich (Sydney, NSW, Australia). Phusion High-Fidelity DNA Polymerase, 1 kb DNA Ladder, and restriction enzymes were from New England Biolabs (Ipswich, MA); Dulbecco's modified Eagle's medium, LipofectAMINE 2000, minimum essential media (MEM) nonessential amino acids, MEM sodium pyruvate, peptone, Opti-MEM PLUS reagent and penicillin streptomycin were from Invitrogen (Mount Waverley, VIC, Australia). Morphine hydrochloride was obtained from GlaxoSmithKline (Melbourne, Australia). Morphine 3-glucoside was synthesized inhouse, as described by Chau et al. (2014). Solvents and other reagents, including acetic acid, acetonitrile, triethylamine, and perchloric acid, were of analytical reagent grade.

Comparative Modeling and MD Simulations. A UGT2B7 homology model (residues 23-469) has been developed in this laboratory based on the X-ray crystal structure of Medicago truncatula UGT85H2 (Protein Data Bank 2PQ6) using SWISS-MODEL (Nair et al., 2015). In silico mutants were generated by substituting Arg259 of the wild-type structure with Ala and Leu (see Results and Discussion) using the mutagenesis tool in PyMOL (PyMOL, Version 2.0; Schrödinger, LLC). MD simulations of the wild-type and mutant UGT2B7 proteins were performed using GROMACS 2019 in conjunction with the GROMOS 54A7 force field (Schmid et al., 2011; Abraham et al., 2015). The simple point charge water model was used to describe the solvent water. Simulations were performed under periodic boundary conditions in a rectangular box. A cut-off of $1.2 \mathrm{~nm}$ was applied to short-range nonbonded interactions, whereas long-range electrostatic interactions were calculated using the particle mesh Ewald algorithm (Darden et al., 1993; Essmann et al., 1995). UDPGlcUA and UGP-Glc were predicted to have charge states of -3 and -2 , respectively, at $\mathrm{pH}$ 7.4, using the Calculator Plugins implemented in ChemAxon (Marvin 16.6.20). Topology parameters for UDP-GlcUA and UGP-Glc were obtained using the Automated Topology Builder and Repository (Malde et al., 2011).

UDP-GlcUA and UDP-Glc were docked into the cofactor binding domain by overlaying the template UDP-deoxy-fluoro-glucose from the VvGT1 crystal structure, as described previously (Meech et al., 2015; Nair et al., 2015). The UDP-Glc/UDP-GlcUA-UGT2B7 complexes were separately placed in a cubic box of simple point charge water with neutralizing counter ions. A steepest-descents minimization followed by a position restraint simulation for 250 picoseconds was performed under a constant number of particles, volume, and temperature (NVT) ensemble. Constant number of particles, pressure, and temperature (NPT) equilibration was performed for 250 picoseconds using weak coupling to maintain pressure isotropically at $1.0 \mathrm{bar}$ at a temperature of $310^{\circ} \mathrm{K}$. A Parrinello-Rahman barostat was used to isotropically regulate pressure along with a Nose'-Hoover thermostat to maintain temperature, ensuring that a true NPT ensemble was sampled (Parrinello and Rahman, 1981; Nosé, 1984; Hoover, 1985). Production MD simulations were conducted for 300 nanoseconds without any restraints. Simulations were performed for UGT2B7 wild-type and mutant (Arg259Ala and Arg259Leu) proteins with bound cofactor (UDP-GlcUA and UDP-Glc).

Polymerase Chain Reaction Site-Directed Mutagenesis and Expression of UGT2B7 Proteins. The wild-type UGT2B7 cDNA in pBluescript II SK (+) was used as the template for site-directed mutagenesis (SDM). Primers employed for mutagenesis are shown in Supplemental Table 1. Polymerase chain reaction (PCR) reactions in 
a total volume of $50 \mu$ l contained $100 \mathrm{ng}$ of DNA template, $250 \mathrm{ng}$ of forward and reverse oligonucleotide primer, $0.2 \mathrm{mM}$ of each deoxyribose NTP, and 1.0 U Phusion High-Fidelity DNA Polymerase. Reaction mixtures were prepared in $5 \times$ Phusion HF reaction buffer. The PCR reaction was performed using a RoboCycler Gradient 96 temperature cycler (Stratagene, La Jolla, CA). Cycling parameters were 3 minutes at $98^{\circ} \mathrm{C}$ for DNA denaturation and activation of the polymerase followed by 18 cycles of 10 seconds at $98^{\circ} \mathrm{C}$ for denaturation, 1 minute at $55^{\circ} \mathrm{C}$ for primer annealing, and 14 minutes at $68^{\circ} \mathrm{C}$ for extension with a final extension of 15 minutes at $68^{\circ} \mathrm{C}$. PCR products were purified using the QIAquick PCR Purification Kit. The PCR product was then digested with $20 \mathrm{U}$ of $D p n I\left(37^{\circ} \mathrm{C}\right.$ for 60 minutes) to cleave the methylated template plasmid and heatinactivated at $80^{\circ} \mathrm{C}$ for 20 minutes. After transformation into $\mathrm{DH} 5 \alpha$ cells and purification, wild-type and mutant UGT2B7 coding sequences were then ligated into the mammalian vector pEF-IRES-puro 5 using the restriction enzymes XhoI and XbaI. All mutations were confirmed on both strands by DNA sequencing using an Applied Biosystems 3130xl Genetic Analyzer with BigDye Terminator v3.1 Chemistry (Applied Biosystems, Foster City, CA). UGT2B7 and wildtype and mutant cDNAs were stably expressed in HEK293T cells as reported by Uchaipichat et al. (2004), and Chau et al. (2014).

Immunoblotting. Cell lysate from HEK293T cells expressing wild-type and mutant UGT2B7 proteins $(50 \mu \mathrm{g})$ was separated by $10 \%$ SDS-polyacrylamide gel electrophoresis and transferred to nitrocellulose $(0.45 \mu \mathrm{m}$; Bio-Rad Laboratories, Inc., CA). Immunodetection of UGT2B7 proteins was performed by probing with rabbit anti-human UGT2B7 (polyclonal) primary antisera (Kerdpin et al., 2009) diluted 1:2000, which was followed by incubation with Peroxidase AffiniPure Donkey Anti-Rabbit IgG (heavy chain + light chain) secondary horseradish peroxidase-conjugated IgG (Jackson ImmunoResearch Laboratories, Inc., PA) diluted 1:4000. Immunoreactivity was detected using the Clarity Western ECL Substrate (Bio-Rad Laboratories, Inc.). Blots were visualized with a Fujifilm LAS-4000 imaging system (Fujifilm Life Sciences, New South Wales, Australia), and band intensities were measured using Image Studio Lite Version 5.2 (LI-COR, Inc., NE). Relative UGT2B7 protein levels represent the mean of quadruplicate measurements. Western blot analysis and activity assays were performed using the same batch of cell lysate.

Morphine 3- and 6-Glycosidation, AZT and 4MU Glucuronidation Assays, and Metabolite Quantification. The method employed was a modification of the procedure for morphine 3- and 6glucuronidation reported by Uchaipichat et al. (2011). Briefly, incubations in a total volume of $200 \mu \mathrm{l}$ contained phosphate buffer $(0.1 \mathrm{M}$, $\mathrm{pH} 7.4), \mathrm{MgCl}_{2}$ (4 mM), recombinant UGTs expressed in HEK293 cells (0.4 mg), morphine (1-10 mM), and UDP-GlcUA or UDP-Glc (5 mM). After 5 minutes preincubation at $37^{\circ} \mathrm{C}$ in a shaking water bath, reactions were initiated by the addition of cofactor (UDP-Glc or UDPGlcUA) and performed for 150 minutes. Reactions were terminated by the addition of perchloric acid (11.6 M; $2 \mu \mathrm{l})$ and cooling on ice for 10 minutes. Samples were centrifuged (5000g for 10 minutes), and a $10-\mu l$ aliquot of the supernatant fraction was analyzed by highperformance liquid chromatography.

Experiments utilizing AZT and 4MU as the aglycones and UDPGlcUA as the cofactor were as described for the morphine glycosidation assay, except for altered protein concentration $(0.3$ and $0.2 \mathrm{mg}$ of HEK293 cell lysate, respectively) and incubation time (60 minutes) (Lewis et al., 2007; Rowland et al., 2007). The respective concentrations of AZT and 4MU in incubations ranged from $0.1-1$ to 0.05-0.7 mM, respectively. Reactions were terminated with $2 \mu \mathrm{l}$ of perchloric acid, and an aliquot ( 15 and $40 \mu \mathrm{l}$, respectively) of the supernatant fraction was analyzed by HPLC. Morphine $3-\beta-D-$ glucuronide, morphine $6-\beta$-D-glucuronide, morphine 3 -glucoside, and 4-methylumbelliferone glucuronide formation was quantified by HPLC as described by Chau et al. (2014), whereas zidovudine glucuronide formation was measured according to Rowland et al. (2007) and Lewis et al. (2007).

\section{Results}

UGT2B7 Homology Model and MDS. A human UGT2B7 homology model was constructed using the X-ray crystal structure of UGT $85 \mathrm{H} 2$ from $M$. truncatula. The model incorporates a GT-B fold that distinguishes the CT and NT domains (Fig. 1A), which is consistent with previously published plant UGT X-ray crystal structures. Similar to the UGT2B7-CT X-ray crystal structure, the CT domain of the model includes a highly conserved secondary structure with a Rossman-type fold, in which seven $\alpha$-helices envelop a single parallel $\beta$-sheet comprising six individual strands with an rmsd of $1.6 \AA$. The signature sequence (residues 356-399) of the CT domain, which is associated with UDP-sugar binding, is shown in Fig. 1B. Like the previous full-length UGT2B7 model developed by Miley et al. (2007), Ser34, His35, and Asp151 are located in close proximity to the signature sequence; for example, the distance between Ser34 and Asn378 is $3.2 \AA$. These residues are thought to function as a serine hydrolase-like catalytic triad (Miley et al., 2007).

NT Domain Amino Acids Contribute to UDP-Sugar Binding and Confer UDP-Sugar Selectivity. MD simulations of the UDP-Glc and UDP-GlcUA bound structures showed Tyr33 and Met37 are located within $5 \AA$ of the uracil moiety of the UDP-sugar (Fig. 2). It has been demonstrated that the $\mathrm{S}$ atom of Met can interact with aromatic (Aro) amino acids. The so-called Met-Aro interactions are generally stronger than hydrophobic interactions, resulting in additional stabilization of 1-1.5 kcal/mol (Valley et al., 2012). It has also been demonstrated that Met can participate in Aro-Met-Aro bridging interactions, which are highly stabilizing and can serve as "recognition" motifs (Weber and Warren 2019). During the simulations of unliganded UGT2B7, it was observed that Tyr33 and Trp356 are located within $5 \AA$ of Met37, and this results in a Tyr33-Met37-Trp356 bridging interaction. Simulations of UGT2B7 in the presence of UDP-Glc and UDP-GlcUA showed two additional bridging interactions involving the uracil ring of the UDP-sugar (namely Tyr33-Met37-uracil and Trp356-Met37-uracil). In addition, there is an edge-to-face aromatic $\pi-\pi$ interaction between Tyr33 and the uracil ring of the UDP-sugar. Furthermore, the Ser34 side chain is flexible and $\mathrm{H}$-bonds with the $\alpha$-phosphate $\mathrm{O} 2$ atom. Simulations show a combination of hydrophobic, polar, and aromatic residues (Ser173, Phe174, and Thr179) positioned within $5 \AA$ of the sugar moiety of both UDP-Glc and UDP-GlcUA, which provides a binding pocket for the polar hydroxyl and hydrophobic - $\mathrm{CH}$ groups of the sugar rings.

A notable difference observed in the MD simulations of the UDP-Glc and UDP-GlcUA bound UGT2B7 complexes was the side-chain conformation of Arg259. In the simulations with UDP-Glc, the Arg259 side chain spontaneously "flipped" away from the UDP-sugar binding site such that the distance between the $\mathrm{O}$ atom of the $-\mathrm{CH}_{2} \mathrm{OH}$ group of UDP-Glc and the $-\mathrm{NH}$ of the guanidinium moiety of arginine equilibrated at a distance of approximately $12 \AA$ (Fig. 3A). Although the simulations showed that the side-chain $-\mathrm{CH}_{2} \mathrm{OH}$ group of glucose is highly flexible and forms transient H-bonds with Ser173, Ser175, Thr179, and Ala377 (backbone -NH atom), H-bonding with Arg259 did not occur during the course of the simulation. In contrast, MD simulations of UDP-GlcUA bound UGT2B7 demonstrated a conformational "locking" of the Arg259 side chain and the carboxylate group of glucuronic 
A

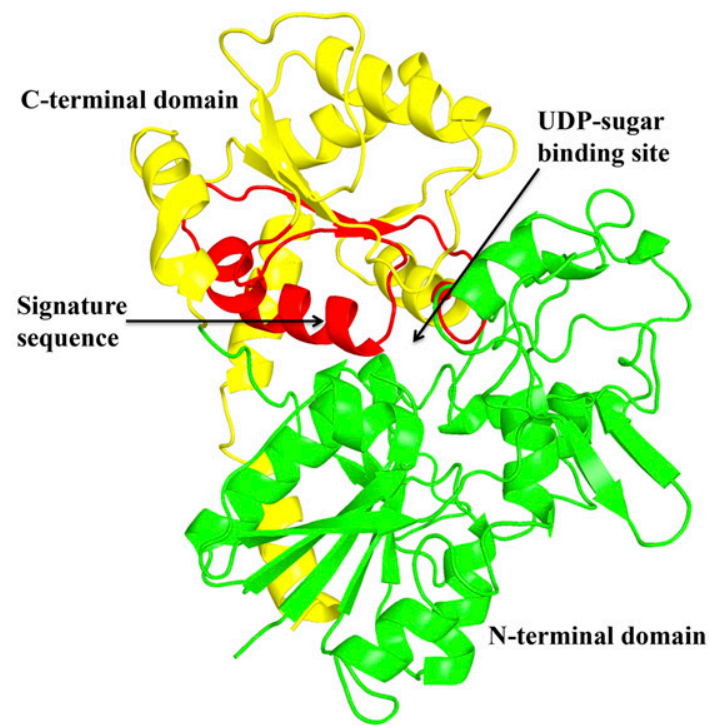

B

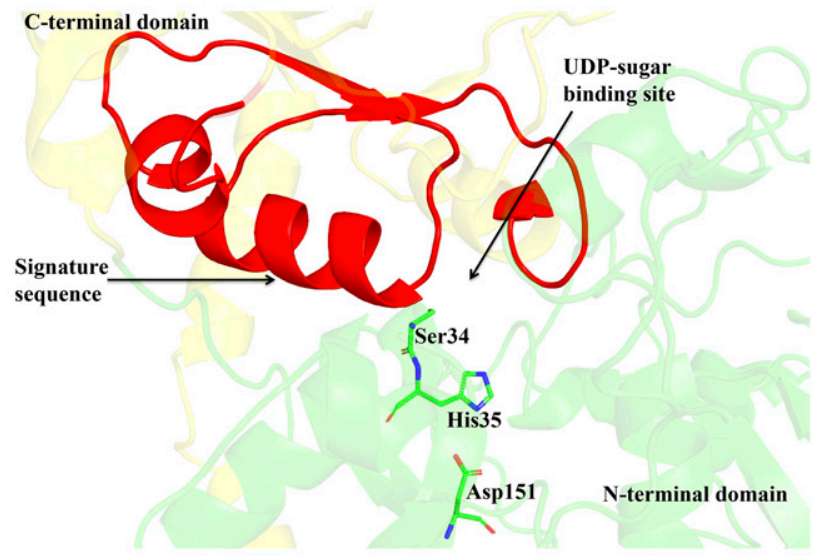

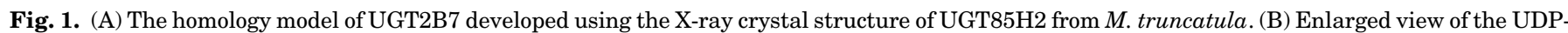

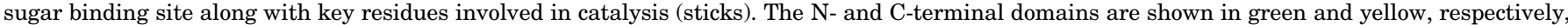
whereas the signature sequence is shown in red.

acid. As shown in Fig. 3B, the distance between the side-chain Arg259 and the carboxylate group fluctuates between the "in" and "out" conformations for the first 100 nanoseconds of the simulation, but beyond 100 nanoseconds the Arg259 side chain remains in a stable locked conformation such that the distance between the $\mathrm{H}$ atom of Arg259 (guanidinium group) and the carboxylate $\mathrm{O}$ atoms is relatively stable at approximately $2.2 \AA$ (Supplemental Movie 1). In this orientation, the Arg259 side chain also H-bonds with the side chain of Asn378, which stabilizes the locked conformation. In the locked orientation, a stable salt bridge forms between the positively charged $\mathrm{N}$ atom of the guanidinium group of Arg259 and the negatively charged carboxylate $\mathrm{O}$ atom of glucuronic acid (Fig. 3C); at equilibrium (>100 nanoseconds), the distance between the two groups is approximately $3.8 \AA$.

UDP-Glc/UDP-GlcUA Binding Site at the CT Domain. UGT2B7-UDP-Glc and UGT2B7-UDP-GlcUA complexes were obtained by docking the cofactors at the binding site near the signature sequence of the CT domain, as described previously (Meech et al., 2015; Nair et al., 2015). MD simulations of both complexes showed a stable binding mode within the cofactor binding domain, which is consistent with the previously identified site predicted by the partial X-ray structure of UGT2B7, the plant X-ray crystal structures, and the homology models based on plant UGT templates (Offen et al., 2006; Miley et al., 2007; Nair et al., 2015).

In particular, simulations of UGT2B7 with bound UDP-Glc indicated a T-shaped aromatic ring stacking $\pi-\pi$ interaction between the side chain of Trp356 and the uracil of UDP-Glc, as predicted for human UGT2B7 and other UGT enzymes (Nair et al., 2015) (Fig. 2A). Furthermore, the uracil ring was found to lie in close proximity to Arg338 such that flexibility in the side chain allows $\mathrm{H}$-bonding with $\mathrm{O} 1$ of the uracil ring. Tyr354, Ile357, Pro358, and Phe371 together form an aromatic hydrophobic "pocket" near the uracil ring. The simulations also demonstrated H-bonding interactions $(\sim 2.5 \AA)$ between the $\mathrm{O}$ atom of the ribose $1^{\prime}-\mathrm{OH}$ and the side chain $-\mathrm{NH}$ of Gln359, whereas the carboxylate group of the Glu382 side chain formed an H-bond ( $\sim 2 \AA$ ) with the 2 -OH group. Likewise, the side chain hydroxyl of Thr373 and nitrogen $\left(\mathrm{N} \varepsilon^{2}\right)$ of His374 H-bond with the $\mathrm{O} 1$ atoms of $\alpha$ - and $\beta$-phosphate. Furthermore, the $\mathrm{O}_{2}$ atoms of $\alpha$ - and $\beta$-phosphate $\mathrm{H}$-bond with Ser311 (both side-chain and backbone atoms) and the backbone atoms of Asn378. The backbone -NH of Gly379 H-bonds with the $\mathrm{O}_{2}$ atom of the $\beta$-phosphate group. MD simulations of UDP-Glc at the UGT2B7 binding site further show an identical H-bonding pattern between Asp398 and Gln399 and the $\mathrm{O}$ atoms of the glucose ring, as predicted previously for UDP-GlcUA (Miley et al., 2007), and H-bonding interactions between the side chain $-\mathrm{NH}$ of Asn402 and the $3^{\prime} \mathrm{OH} /$ $4^{\prime} \mathrm{OH}$ groups of the glucose ring. The residues at positions 398 , 399 , and 402 lie on the C $\alpha 5$ helix of the CT domain. Simulations of the unliganded and the cofactor bound UGT2B7 show that the $\mathrm{C} \alpha 5$ helix region is relatively rigid compared with the loop region associated with residues (e.g., Arg338, Trp356, Ile357) that interact with the uracil ring of the UDP-sugar. This less-malleable region of the CT domain may be an essential recognition site for the UDP-sugars as mutation of Asp398, Gln399, and Asn402 completely abolishes glycosidation activity (Miley et al., 2007; Chau et al., 2014).

As alluded to above, MD simulations of UDP-GlcUA bound to UGT2B7 show similar interactions between the uracil ring and Arg338, Trp356, and Leu357 to those noted for UDP-Glc (Fig. 1B). Several of the differences observed in the MD simulations of UDP-GlcUA are associated with the positioning of side-chain atoms of some residues located near the ribose ring, phosphate groups, and glucuronic acid moiety. Specifically, Glu382 forms a bidentate $\mathrm{H}$-bond with both -OH groups of ribose $(\sim 2 \AA)$, whereas Gln359 moderately H-bonds $(\sim 3 \AA)$ with the $\mathrm{O}$ atoms of the $1^{\prime}-\mathrm{OH}$ group. His374 repositions to $\mathrm{H}$-bond with the $\mathrm{O} 1$ atom of $\beta$-phosphate and $2^{\prime} \mathrm{OH} / 3^{\prime} \mathrm{OH}$ of glucuronic acid, whereas Gln399 H-bonds with $3^{\prime} \mathrm{OH} / 4^{\prime} \mathrm{OH}$ glucuronic acid. The glucuronic acid moiety positions such that Asp398 makes electrostatic contact with 4' $\mathrm{OH}$ instead of H-bonding as observed in the UDP-Glc simulations. This 


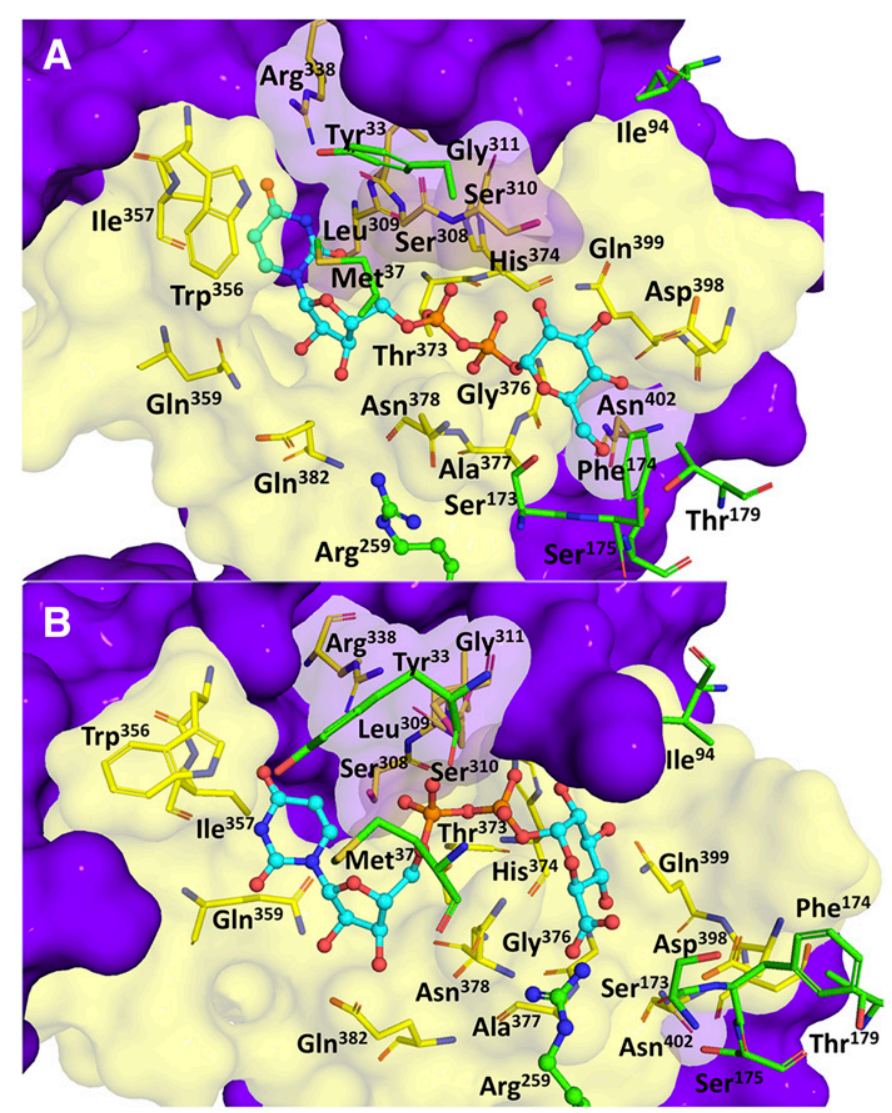

Fig. 2. MD simulations of cofactor bound UGT2B7. (A) Binding mode of UDP-Glc, and (B) binding mode of UDP-GlcUA showing the average position of side-chain residues. The CT domain is shown in purple (surface), the signature sequence is shown in yellow (transparent surface), and amino acids important for cofactor binding are shown as yellow sticks (C atoms). The NT domain residues are shown as green sticks (C atoms). O, $\mathrm{N}, \mathrm{S}$, and $\mathrm{P}$ atoms are shown in red, blue, yellow, and orange, respectively.

repositioning of the sugar ring is most likely due to the electrostatic repulsion between the carboxylate groups.

Site-Directed Mutagenesis Confirms Arg259 as an Important Residue for the Selective Binding of UDP-GlcUA. To verify the importance of $\mathrm{H}$-bond and saltbridge formation between Arg259 and the carboxylate group of glucuronic acid in cofactor binding and selectivity, two mutants were generated: Arg259Leu and Arg259Ala. Leu has a neutral hydrophobic side chain but similar volume to Arg, and substitution with Ala is a common approach for investigating the importance of a residue in ligand binding. Wild-type UGT2B7 and the Arg259Ala and Arg259Leu mutants were stably expressed in HEK293T cells. Analysis of the densitometry data from quadruplicate blots provided mean $( \pm$ S.D.) relative densities (compared with wild-type UGT2B7) of $0.16 \pm 0.03$ (Arg259Ala) and $0.39 \pm 0.04$ (Arg259Leu) (Supplemental Fig. 1). Human liver microsomes were used as the positive control, whereas untransfected HEK293 lysate served as the negative control. Activities of UGT2B7 and the Arg259Ala and Arg259Leu mutants were determined for AZT, 4MU, and morphine with both UDP-Glc and UDP-GlcUA (5 mM) as the cofactors (Fig. 4). Activities normalized for relative expression were determined at three substrate (aglycone) concentrations that spanned the known $\mathrm{K}_{\mathrm{m}}$ values for AZT, $4 \mathrm{MU}$, and morphine glucuronidation by wild-type UGT2B7 (Stone et al., 2003; Uchaipichat et al., 2006; Lewis et al., 2007; Chau et al., 2014 and unpublished data). The Ala and Leu substitutions abolished the glucuronidation of all three substrates. By contrast, morphine 3-glucosidation was unaffected by the Arg259-to-Leu substitution, although the

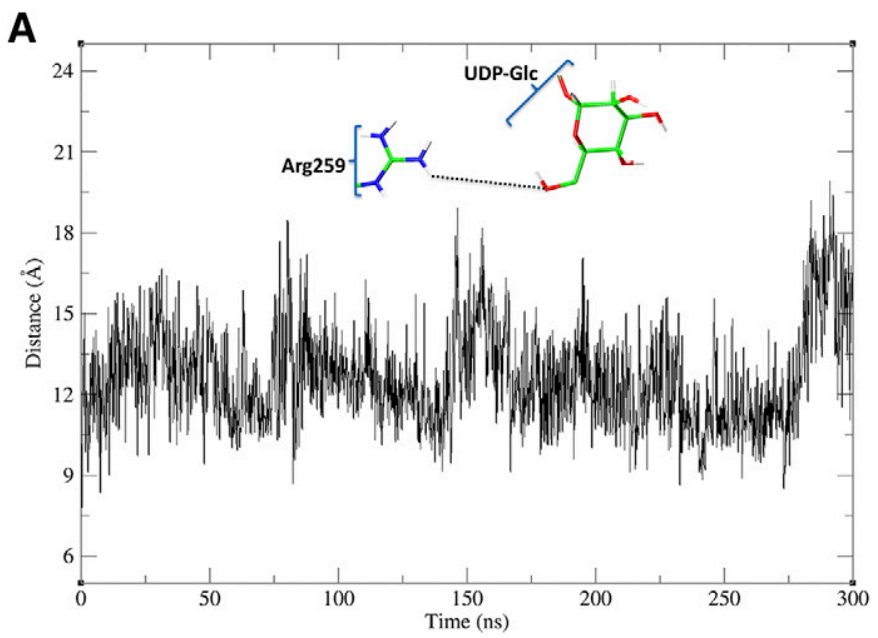

B

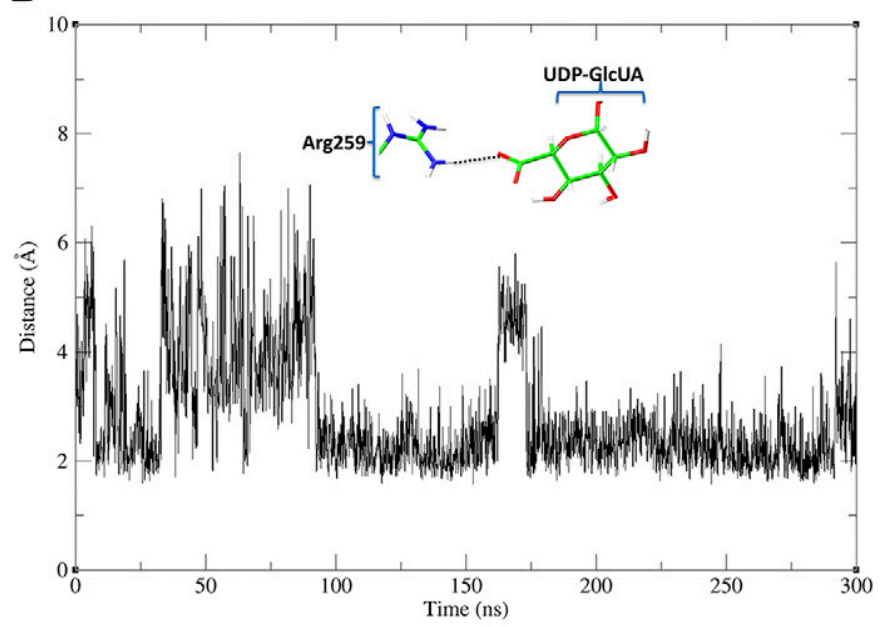

C

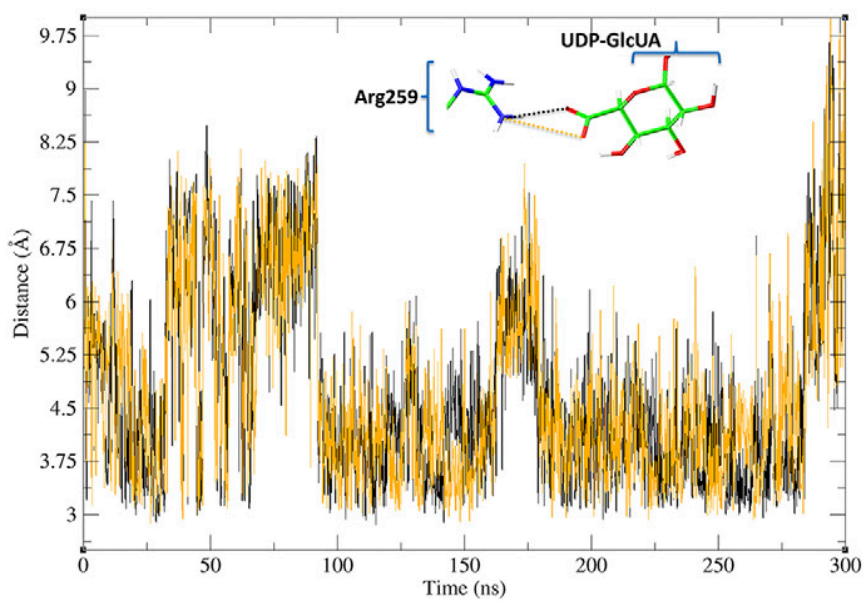

Fig. 3. (A) Distance between the side chain of Arg259 (-NH) and $-\mathrm{CH}_{2} \mathrm{OH}$ (O) of UDP-Glc. (B) Distance between the side chain of Arg259 (-NH) and the $-\mathrm{COO}^{-}(\mathrm{O})$ of UDP-GlcUA. (C) Distance between the $\mathrm{N}$ atom of the Arg259 side chain and the $-\mathrm{COO}^{-}$(O atoms) of UDP-GlcUA. 
A

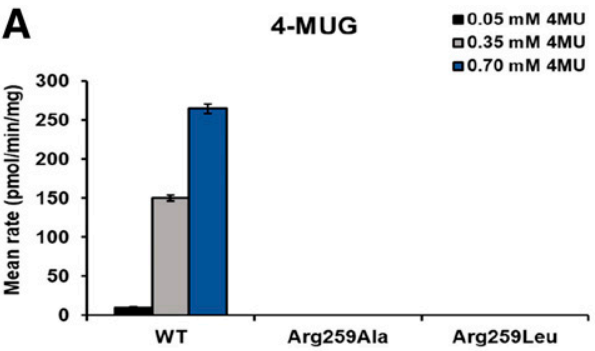

C
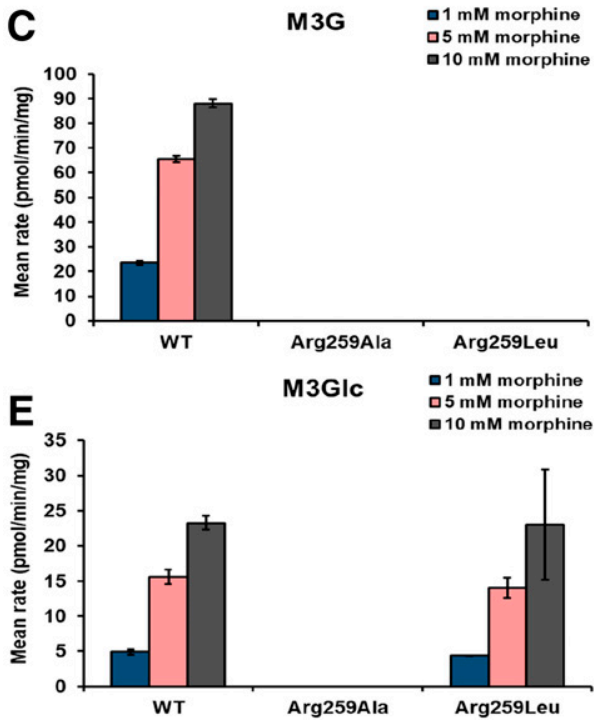

B

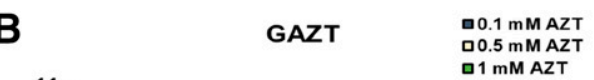

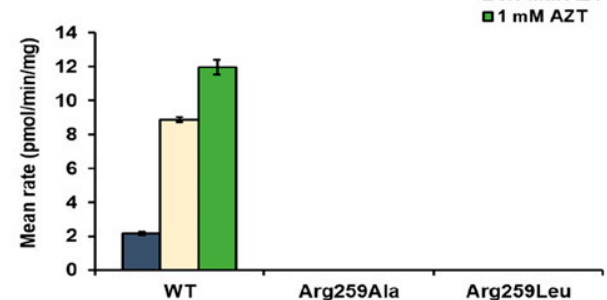

D

M6G

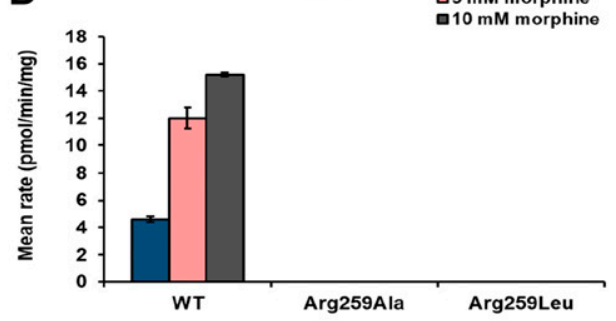

Fig. 4. 4MU, AZT, and morphine glucuronidation (A-D) and morphine glucosidation (E) activities of wild-type UGT2B7 and the Arg259Ala and Arg259Leu mutants. Activities shown as mean \pm S.D. $(n=4)$. The absence of bars indicates no measurable activity. GAZT, zidovudine glucuronide; M3G, morphine $3-\beta$-D-glucuronide; M6G, morphine 6- $\beta$-D-glucuronide; 4-MUG, 4-methylumbelliferone glucuronide; M3Glc, morphine 3-glucoside; WT, wild type.
Arg259Ala mutant lacked morphine 3-glucosidation activity. The absence of an effect of the Arg259Leu substitution on morphine 3-glucuosidation accords well with the MD simulations that showed Arg259 did not contribute to UDP-Glc binding.

MD simulations of the Arg259Leu mutant with bound UDPGlcUA showed an altered binding conformation that impacted its interactions with both NT and CT residues (Supplemental Fig. 2). For example, the uracil moiety of UDP-GlcUA bound close to Tyr33 in the wild-type UGT2B7 structure, whereas glucuronic acid bound close to this residue in the Arg259Leu mutant. Furthermore, the interactions of UDP-GlcUA with His374, Gln399, and Asn398 observed in UGT2B7 were absent in the Arg259Leu mutant. Although the Arg259Leu mutant catalyzed morphine 3-glucosidation, MD simulations of Arg259Leu with bound UDP-Glc showed no direct contact between Leu259 and the cofactor, suggesting that bulkiness at this position is essential for maintaining the integrity of the cofactor binding site. This conclusion is in agreement with the observation that UGT3A1 catalyzes the glucosidation but not glucuronidation of a number of chemicals (Meech et al., 2012). UGT3A1 has Asn, which has a similar volume to Arg, at position 259 (UGT2B7 numbering) (Supplemental Fig. 3). By contrast, MD simulations of the Arg259Ala mutant bound with either UDPGlcUA or UDP-Glc showed conformational changes of residues, leading to the loss of nearly all interactions between key binding residues (e.g., Tyr33, Met37, Trp356, Glu382) and UDP-GlcUA and resulting in disorganization of the cofactor binding domain, which is suggestive of an unstable protein. The latter observation is consistent with the low expression of the Arg259Ala mutant determined from Western blots.

\section{Discussion}

UGT2B7 contributes to the elimination of numerous drugs, nondrug xenobiotics, and endogenous compounds. The major metabolic pathway catalyzed by UGT2B7 is glucuronidation, which utilizes UDP-GlcUA as the cofactor. However, as noted previously, this enzyme additionally catalyzes the glucosidation of several substrates, including morphine (Chau et al., 2014 and references therein). Our previous analysis of a UGT2B7 homology model suggested that, in addition to the CT domain, residues of the NT domain also contribute to UDP-sugar binding and hence potentially to sugar selectivity. On the basis of our analysis of the homology model (Nair et al., 2015) and on sequence conservation data (RadominskaPandya et al., 2010), it was postulated that Arg259 may be involved in the selective recognition of UDP-GlcUA. Thus, MD simulations together with SDM and enzyme kinetic studies were performed with wild-type UGT2B7 and the Arg259Ala and Arg259Leu mutants in the absence and presence of bound cofactor (UDP-GlcUA/UDP-Glc) to characterize the relative contributions of the CT and NT domains to cofactor binding and selectivity.

Experimental techniques, such as SDM and X-ray crystallography, have been used previously to gain insights into the structure-function relationships of UGT enzymes. However, these approaches have only provided limited understanding of the contributions of molecular flexibility and dynamic motions of individual residues to cofactor binding and selectivity. By 
contrast, MD simulations, in combination with experimental approaches, has provided increased knowledge of protein structure-function relationships for several biologic systems, including drug-metabolizing cytochrome P450 enzymes (Nair et al., 2016). Importantly, MD simulations provide a means to model the plasticity of proteins at an atomic level (Nair and Miners, 2014; Nair et al., 2016). Only two MD simulation studies that investigated ligand binding to UGT proteins (namely UGT1A6 and UGT1A9/10) have been reported to date (Tripathi et al., 2016; Smith et al., 2020). Neither of these studies provided experimental verification of residues predicted to be involved in UDP-sugar binding nor insights into the residues that are essential for cofactor selectivity. Furthermore, both studies employed short simulation times (50 nanoseconds), which may not be adequate for detailed sampling of conformational space and accurate prediction of protein-ligand interactions. Here, we used long simulation times to generate hypotheses of UDP-sugar selectivity that were tested using SDM and kinetic studies.

Data from MDS highlighted the presence of a handful of residues in the NT domain that contribute to UDP-sugar binding and selectivity. As noted above, Tyr33 was found to be important and formed aromatic $\pi-\pi$ interaction between Tyr33 and the uracil ring of the UDP-sugar. A previous SDM study from this laboratory demonstrated that the Tyr33Leu mutant lacked glucosidation and glucuronidation activity, whereas the Tyr33Phe mutant retained glycosidation activity (Chau et al., 2014). This is consistent with the importance of aromaticity contributed by Tyr33 for UDPsugar binding.

Furthermore, MDS demonstrated that the side chain of Arg259 forms a stable salt-bridge interaction with the carboxylate O group of UDP-GlcUA. Salt bridges, which occur between oppositely charged groups, are the strongest noncovalent interactions and are known to contribute significantly to ligand binding (e.g., Wester et al., 2004; Miyamoto et al., 2011). For instance, the positively charged Arg108 in the active site of CYP2C9 is a key residue in the recognition of the negatively charged weak acids (e.g., carboxylic acids) that are metabolized by this enzyme (Wester et al., 2004). The occurrence of both H-bonding and salt-bridge formation between the UDP-GlcUA and Arg259, which is absent with UDP-Glc, would appear to be the critical factor responsible for the higher binding affinity of UDP-GlcUA to UGT2B7 and is also consistent with glucuronidation being the dominant metabolic pathway for substrates of this enzyme. Moreover, the contribution of NT residues to UDP-GlcUA binding provides a plausible explanation why cofactor does not cocrystallize with the CT domain alone (Miley et al., 2007).

Sequence analysis of human UGT enzymes shows that Arg259 is a conserved residue in UGT1 and UGT2 family proteins (Supplemental Fig. 3). Consistent with this observation, all members of the UGT1 and UGT2 families use UDPGlcUA as a cofactor and hence form glucuronide conjugates. The recent UGT1A6 model reported by Smith et al. (2020) showed the presence of Arg256 (equivalent to Arg259 in UGT2B7) in the cofactor binding site (Supplemental Fig. 3). However, this residue interacted with the phosphate group of UDP-GlcUA. Notably, the simulations by Smith et al. (2020) were performed for 50 nanoseconds and did not reach equilibrium, as the rmsd increased with time. In contrast, our simulations show that the rmsd of the $\mathrm{C}_{\alpha}$ atoms of UGT2B7 in the presence of UDP-Glc $(\sim 2.2 \AA)$ and UDP-GlcUA $(\sim 2 \AA)$ stabilized after approximately 50 and 100 nanoseconds, respectively (Supplemental Fig. 4), suggesting longer simulation times are essential for attaining equilibrium. Since the stability in the binding interactions involving Arg only occurred after 100 nanoseconds (Fig. 3, B and C), crucial side-chain conformations may not be evident with shorter simulations.

In contrast to UGT1 and UGT2 family enzymes, Arg259 (UGT2B7 numbering) is not conserved in UGT3 and UGT8 proteins, in which the equivalent position is substituted with Asn and Cys, respectively. UGT3A1, UGT3A2 and UGT8 do not use UDP-GlcUA (Meech et al., 2012, 2015), further supporting the critical role of the conserved Arg at position 259 (UGT2B7 numbering) in UDP-GlcUA binding.

Miley et al. (2007) solved the first X-ray crystal structure of a human UGT2B7, namely the CT domain of UGT2B7. However, cocrystallization of the cofactor UDP-GlcUA within the partial UGT2B7 structure was not achieved. Hence, a plant X-ray structure with a bound cofactor (UDP-2-deoxy2-fluro glucose) was used to predict the binding of UDP-GlcUA within the UGT2B7 protein. The model predicted a ringstacking interaction between the conserved Trp356 and uracil, as observed in the plant UGT structure; packing of Gln359 adjacent to the uracil ring; H-bonding of Glu382 to the ribose 2'-OH group; formation of a salt bridge between His374 and the negative charge of the $\beta$-phosphate; H-bonding between Asn378 and the $\alpha$-phosphate; H-bonding of the Thr373 side chain with the di-phosphate group; and H-bonding of Asp398 and Gln399 with the $\mathrm{O}_{3}{ }^{\prime} / \mathrm{O}_{4}{ }^{\prime}$ and $\mathrm{O}_{2}{ }^{\prime} / \mathrm{O}_{3}{ }^{\prime}$ atoms of glucuronic acid, respectively. Based on these observations and the results of SDM experiments, Miley et al. (2007) concluded that disruption of the interactions with either the di-phosphate or glucuronic acid moieties of UGT2B7 abolished activity, whereas disruption of interactions with uracil reduced activity to a moderate extent. Chau et al. (2014) developed a homology model of UGT2B7 based on multiple template structures (UDP-glucose flavonoid 3-O-glucosyltransferase, barrel medic UDP-glucose flavonoid/triterpene glucosyltransferase UGT71G1, and the cofactor binding domain of UGT2B7) and employed automated docking to investigate UDP-sugar selectivity. The model predicted identical binding modes for the UDP moiety of UDP-Glc and UDP-GlcUA but differences in the residues involved in the binding of the sugar moieties. In particular, Asp398 and Glu399 interacted with hydroxyl groups of the glucose, whereas Asn402 and Tyr33 H-bonded with the glucuronic acid moiety of UDP-GlcUA.

There are no X-ray crystal structures of a UDP-GlcUA bound mammalian UGT protein, and the currently available models are based on the assumption that UDP-Glc and UDPGlcUA interact identically with residues in the cofactor binding site (Miley et al., 2007). Notably, the existing models that predict the UGT-UDP-GlcUA interactions are based on the analysis of plant UGT X-ray structures, in which the cofactor is either UDP-Glc or UDP-Glc analogs. Furthermore, UGT2B7 models based on docking UDP-GlcUA in the static protein do not provide a clear basis for UDP-sugar binding or selectivity (Chau et al., 2014). Overall, our simulations demonstrate that the binding interactions of UDP-Glc and UDP-GlcUA at the CT domain, although not identical, are very similar with only subtle variations involving the adaptive repositioning of residues within the binding site for cofactor recognition. 
Previous studies that used static models that predicted Tyr33, Asn378, and Asn402 of UGT2B7 were important for UDP-sugar selectivity, and as noted above, mutagenesis of these amino acids led to loss of all glycosidation activity (Miley et al., 2007; Chau et al., 2014). As described above, simulations demonstrated that Tyr33 is located close to the uracil ring of the UDP-sugar, whereas Asn402 interacts with the -OH group of the sugar rings with no direct interaction with the $-\mathrm{CO}_{2}^{-}$or $-\mathrm{CH}_{2} \mathrm{OH}$ groups of UDP-GlcUA or UDP-Glc, respectively. This suggests that both residues may be important for UDP-sugar binding but not sugar selectivity. Furthermore, as noted in the simulations with bound UDP-GlcUA, Arg259 H-bonds with Asn378, allowing the Arg side chain to form a stable salt bridge with the carboxylate group of UDPGlcUA. The SDM results reported here demonstrated that the Arg259Leu mutant catalyzed the glucosidation but not glucuronidation of morphine, indicating that Arg259 (and not Asn378) is the critical residue for the selective binding of UDPGlcUA over UDP-Glc.

\section{Conclusions}

This is the first structure-function study of a UGT enzyme that has employed MD simulations for hypothesis generation with subsequent experimental verification. The study demonstrated the importance of structural flexibility and the physicochemical properties of amino acids in the selective binding of UDP-sugars within UGT2B7 active site. In particular, Arg259, which is located in the NT, was shown to be critical for binding the carboxylate group of glucuronic acid via $\mathrm{H}$-bonding and salt-bridge formation. The study further demonstrated that conformational equilibrium and stable interactions between UDP-GlcUA and cofactor binding site residues were only achieved with simulation times $>100$ nanoseconds, suggesting that shorter simulations may present incomplete sampling, and crucial conformational rearrangements may be overlooked.

\section{Acknowledgments}

P.C.N. acknowledges Flinders University for Early Career Research Funding.

\section{Authorship Contributions}

Participated in research design: Nair, Chau, McKinnon, Miners.

Conducted experiments: Nair, Chau.

Contributed new reagents or analytic tools: Nair, McKinnon, Miners.

Performed data analysis: Nair, Chau, McKinnon, Miners.

Wrote or contributed to the writing of the manuscript: Nair, Chau, McKinnon, Miners.

\section{References}

Abraham MJ, Murtola T, Schulz R, Páll S, Smith JC, Hess B, and Lindahl E (2015) GROMACS: high performance molecular simulations through multi-level parallelism from laptops to supercomputers. SoftwareX 1-2:19-25.

Chau N, Elliot DJ, Lewis BC, Burns K, Johnston MR, Mackenzie PI, and Miners JO (2014) Morphine glucuronidation and glucosidation represent complementary metabolic pathways that are both catalyzed by UDP-glucuronosyltransferase 2B7: kinetic, inhibition, and molecular modeling studies. J Pharmacol Exp Ther $\mathbf{3 4 9}$ $126-137$.

Court MH, Krishnaswamy S, Hao Q, Duan SX, Patten CJ, Von Moltke LL, and Greenblatt DJ (2003) Evaluation of 3'-azido-3'-deoxythymidine, morphine, and codeine as probe substrates for UDP-glucuronosyltransferase 2B7 (UGT2B7) in human liver microsomes: specificity and influence of the UGT2B $7 * 2$ polymorphism. Drug Metab Dispos 31:1125-1133.

Darden T, York D, and Pedersen L (1993) Particle mesh Ewald: an N.log(N) method for Ewald sums in large systems. J Chem Phys 98:10089.
Essmann U, Perera L, Berkowitz ML, Darden T, Lee H, and Pedersen LG (1995) A smooth particle mesh Ewald method. J Chem Phys 103:8577-8593.

Feingold DS and Franzen JS (1981) Pyridine nucleotide-linked four-electron transfer dehydrogenases. Trends Biochem Sci 6:103-105.

Hoover WG (1985) Canonical dynamics: equilibrium phase-space distributions. Phys Rev A Gen Phys 31:1695-1697.

Kerdpin O, Mackenzie PI, Bowalgaha K, Finel M, and Miners JO (2009) Influence of $\mathrm{N}$-terminal domain histidine and proline residues on the substrate selectivities of human UDP-glucuronosyltransferase 1A1, 1A6, 1A9, 2B7, and 2B10. Drug Metab Dispos 37:1948-1955.

Lewis BC, Mackenzie PI, Elliot DJ, Burchell B, Bhasker CR, and Miners JO (2007) Amino terminal domains of human UDP-glucuronosyltransferases (UGT) 2B7 and 2B15 associated with substrate selectivity and autoactivation. Biochem Pharmacol 73:1463-1473.

Mackenzie PI, Bock KW, Burchell B, Guillemette C, Ikushiro S, Iyanagi T, Miners JO, Owens IS, and Nebert DW (2005) Nomenclature update for the mammalian UDP glycosyltransferase (UGT) gene superfamily. Pharmacogenet Genomics 15: $677-685$

Mackenzie PI, Rogers A, Treloar J, Jorgensen BR, Miners JO, and Meech R (2008) Identification of UDP glycosyltransferase 3A1 as a UDP N-acetylglucosaminyltransferase. $J$ Biol Chem 283:36205-36210.

Malde AK, Zuo L, Breeze M, Stroet M, Poger D, Nair PC, Oostenbrink C, and Mark AE (2011) An automated force field topology builder (ATB) and repository: version 1.0. J Chem Theory Comput 7:4026-4037.

Meech R, Hu DG, McKinnon RA, Mubarokah SN, Haines AZ, Nair PC, Rowland A, and Mackenzie PI (2019) The UDP-glycosyltransferase (UGT) superfamily: new members, new functions, and novel paradigms. Physiol Rev 99:1153-1222.

Meech R, Mubarokah N, Shivasami A, Rogers A, Nair PC, Hu DG, McKinnon RA and Mackenzie PI (2015) A novel function for UDP glycosyltransferase 8: galactosidation of bile acids. Mol Pharmacol 87:442-450

Meech R, Rogers A, Zhuang L, Lewis BC, Miners JO, and Mackenzie PI (2012) Identification of residues that confer sugar selectivity to UDP-glycosyltransferase 3A (UGT3A) enzymes. J Biol Chem 287:24122-24130.

Miley MJ, Zielinska AK, Keenan JE, Bratton SM, Radominska-Pandya A, and Redinbo MR (2007) Crystal structure of the cofactor-binding domain of the human phase II drug-metabolism enzyme UDP-glucuronosyltransferase 2B7. J Mol Biol 369:498-511.

Miners JO and Mackenzie PI (1991) Drug glucuronidation in humans. Pharmacol Ther 51:347-369.

Miners JO, Mackenzie PI, and Knights KM (2010) The prediction of drugglucuronidation parameters in humans: UDP-glucuronosyltransferase enzymeselective substrate and inhibitor probes for reaction phenotyping and in vitro-in vivo extrapolation of drug clearance and drug-drug interaction potential. Drug Metab Rev 42:196-208.

Miners JO, Smith PA, Sorich MJ, McKinnon RA, and Mackenzie PI (2004) Predicting human drug glucuronidation parameters: application of in vitro and in silico modeling approaches. Annu Rev Pharmacol Toxicol 44:1-25.

Miyamoto Y, Banno Y, Yamashita T, Fujimoto T, Oi S, Moritoh Y, Asakawa T, Kataoka O, Yashiro H, Takeuchi K, et al. (2011) Discovery of a 3-pyridylacetic acid derivative (TAK-100) as a potent, selective and orally active dipeptidyl peptidase IV (DPP-4) inhibitor. J Med Chem 54:831-850.

Nair PC, McKinnon RA, and Miners JO (2016) Cytochrome P450 structure-function: insights from molecular dynamics simulations. Drug Metab Rev 48:434-452.

Nair PC, Meech R, Mackenzie PI, McKinnon RA, and Miners JO (2015) Insights into the UDP-sugar selectivities of human UDP-glycosyltransferases (UGT): a molecular modeling perspective. Drug Metab Rev 47:335-345.

Nair PC and Miners JO (2014) Molecular dynamics simulations: from structure function relationships to drug discovery. In Silico Pharmacol 2:4.

Nosé S (1984) A unified formulation of the constant temperature molecular dynamics methods. J Chem Phys 81:511.

Offen W, Martinez-Fleites C, Yang M, Kiat-Lim E, Davis BG, Tarling CA, Ford CM, Bowles DJ, and Davies GJ (2006) Structure of a flavonoid glucosyltransferase reveals the basis for plant natural product modification. EMBO J 25 1396-1405.

Parrinello M and Rahman A (1981) Polymorphic transitions in single crystals: a new molecular dynamics method. J Appl Phys 52:7182-7190.

Radominska-Pandya A, Bratton SM, Redinbo MR, and Miley MJ (2010) The crystal structure of human UDP-glucuronosyltransferase 2B7 C-terminal end is the first mammalian UGT target to be revealed: the significance for human UGTs from both the 1A and 2B families. Drug Metab Rev 42:133-144.

Radominska-Pandya A, Czernik PJ, Little JM, Battaglia E, and Mackenzie PI (1999) Structural and functional studies of UDP-glucuronosyltransferases. Drug Metab Rev 31:817-899.

Radominska-Pandya A, Little JM, and Czernik PJ (2001) Human UDP-glucuronosyltransferase 2B7. Curr Drug Metab 2:283-298.

Rowland A, Gaganis P, Elliot DJ, Mackenzie PI, Knights KM, and Miners JO (2007) Binding of inhibitory fatty acids is responsible for the enhancement of UDPglucuronosyltransferase $2 \mathrm{~B} 7$ activity by albumin: implications for in vitro-in vivo extrapolation. J Pharmacol Exp Ther 321:137-147.

Schmid N, Eichenberger AP, Choutko A, Riniker S, Winger M, Mark AE, and van Gunsteren WF (2011) Definition and testing of the GROMOS force-field versions 54A7 and 54B7. Eur Biophys J 40:843-856.

Smith AD, Page BDG, Collier AC, and Coughtrie MWH (2020) Homology modeling of human uridine-5'-diphosphate-glucuronosyltransferase 1A6 reveals insights into factors influencing substrate and cosubstrate binding. ACS Omega 5 $6872-6887$

Stingl JC, Bartels H, Viviani R, Lehmann ML, and Brockmöller J (2014) Relevance of UDP-glucuronosyltransferase polymorphisms for drug dosing: a quantitative systematic review. Pharmacol Ther 141:92-116.

Stone AN, Mackenzie PI, Galetin A, Houston JB, and Miners JO (2003) Isoform selectivity and kinetics of morphine 3- and 6-glucuronidation by human 
udp-glucuronosyltransferases: evidence for atypical glucuronidation kinetics by UGT2B7. Drug Metab Dispos 31:1086-1089.

Tripathi SP, Prajapati R, Verma N, and Sangamwar AT (2016) Predicting substrate selectivity between UGT1A9 and UGT1A10 using molecular modelling and molecular dynamics approach. Mol Simul 42:270-288.

Uchaipichat V, Mackenzie PI, Guo X-H, Gardner-Stephen D, Galetin A, Houston JB, and Miners JO (2004) Human udp-glucuronosyltransferases: isoform selectivity and kinetics of 4-methylumbelliferone and 1-naphthol glucuronidation, effects of organic solvents, and inhibition by diclofenac and probenecid. Drug Metab Dispos 32:413-423.

Uchaipichat V, Raungrut P, Chau N, Janchawee B, Evans AM, and Miners JO (2011) Effects of ketamine on human UDP-glucuronosyltransferases in vitro predict potential drug-drug interactions arising from ketamine inhibition of codeine and morphine glucuronidation. Drug Metab Dispos 39:1324-1328.

Uchaipichat V, Winner LK, Mackenzie PI, Elliot DJ, Williams JA, and Miners JO (2006) Quantitative prediction of in vivo inhibitory interactions involving glucuronidated drugs from in vitro data: the effect of fluconazole on zidovudine glucuronidation. Br J Clin Pharmacol 61:427-439.
Valley CC, Cembran A, Perlmutter JD, Lewis AK, Labello NP, Gao J, and Sachs JN (2012) The methionine-aromatic motif plays a unique role in stabilizing protein structure. J Biol Chem 287:34979-34991.

Weber DS and Warren JJ (2019) The interaction between methionine and two aromatic amino acids is an abundant and multifunctional motif in proteins. Arch Biochem Biophys 672:108053.

Wester MR, Yano JK, Schoch GA, Yang C, Griffin KJ, Stout CD, and Johnson EF (2004) The structure of human cytochrome P450 2C9 complexed with flurbiprofen at 2.0-A resolution. J Biol Chem 279:35630-35637.

Zhang L, Zhu L, Qu W, Wu F, Hu M, Xie W, Liu Z, and Wang C (2020) Insight into tartrate inhibition patterns in vitro and in vivo based on cocrystal structure with UDP-glucuronosyltransferase 2B15. Biochem Pharmacol 172:113753.

Address correspondence to: Dr. Pramod C. Nair, Department of Clinical Pharmacology, Flinders University College of Medicine and Public Health, Flinders Medical Centre, GPO Box 2100, Adelaide, SA 5001, Australia. E-mail: pramod.nair@flinders.edu.au 